\title{
Approximated Solutions of Linear Quadratic Fractional Optimal Control Problems
}

\author{
S. SORADI ZEID, M. YOUSEFI AND M. YOUSEFI
}

\begin{abstract}
In this study we apply the Adomian decomposition method (ADM) to approximate the solution of fractional optimal control problems (FOCPs) where the dynamic of system is a linear control system with constant coefficient and the cost functional is defined in a quadratic form. First we stated the necessary optimality conditions in a form of fractional two point boundary value problem (TPBVP), then the ADM is used to solve the resulting fractional differential equations (FDEs). Some examples are provided to demonstrate the validity and applicability of the proposed method.
\end{abstract}

Mathematics Subject Classification 2010: 65D25, 34A08, 26A33, 49M27

Additional Key Words and Phrases: Adomian decomposition method, Fractional optimal control problem, Fractional two point boundary value problem, Fractional order differential equations.

\section{INTRODUCTION}

In the last decades, fractional calculus (FC) has provided more accurate models in miscellaneous fields of science and engineering. Many mathematicians and applied researchers have tried to model real processes using the fractional calculus. Nigmatullin and Nelson described complex systems in terms of fractional kinetics in [33]. Jesus, Machado and Cunha analyzed the fractional order dynamics in botanical electrical impedances [25; 26]. Petrovic, Spasic and Atanackovic developed a fractional order mathematical model of a human root dentin [37]. In biology, it has been deduced that the membranes of cells of biological organism have fractional order electrical conductance [16] and then are classified in groups of non-integer order models.

The reason of using FDEs is that they are naturally more accurate than integer differential equations to describe the dynamic behavior of many real world [23]. Also, they are closely related to fractals, which are abundant in biological systems [8]. Therefore, it is important to develop formulations and analytical schemes for solving FEDs. Considerable works have been done in this area like ADM [38; 30; $17 ; 34 ; 15 ; 18]$, finite difference method [31], Variational iteration method (VIM) 
[24; 19], Operational matrix method [39], Homotopy analysis method [36; 1], generalized differential transform method [35; 29], finite element method [27], fractional differential transform method $[2 ; 21]$ and references therein.

While the FOCPs that are a subclass of optimal control problems whose dynamics are described by FDEs, have been achieved a growing rate in publications in the area of FC $[43 ; 14 ; 40]$. For that reason, there has been significant interest in developing numerical schemes for their solution. Some numerical methods for solving some type of FOCPs can be seen in $[3 ; 4 ; 13 ; 32]$. A great deal of interest has been recently, focused on the application of ADM to solve a wide variety of these problems. Work on this method first proposed by [5] where the authors have achieved a solution for problem in form of a series whose terms are determined by a recursive relationship using the Adomian polynomials without any need for linearization or discretization or any other restrictive assumption that may change the physical behavior of the model. There also exist other numerical simulations for this purpose, such as $[6 ; 7 ; 20 ; 42]$.

The main aim of this work is to develop a reliable modification of ADM (MADM) to solve a class of FOCPs approximately. For this purpose, we used necessary optimality conditions, to reduce this FOCP to a system of FDEs. Then, we extend the MADM to obtain some new iterative formulas for solving this system of FDEs that is simpler and easier to use.

The outline of this study is as follows: In Section 2, we introduce some definitions and necessary preliminaries for fractional calculus and briefly recall the ADM method. In Section 3, we propose a new analytic approximate method based on ADM for solving a class of FOCPs. In Section 4, Numerical simulations are presented to demonstrate the accuracy of the proposed methods. Finally, we present a brief summary in Section 5 .

\section{PRELIMINARY CONSIDERATIONS}

In this section, we present some notations, definitions and preliminary facts of the fractional calculus theory which will be used further in this work.

\subsection{Fractional Calculus}

There are several approaches to the generalization of the notion of differentiation to fractional orders e.g. Riemann-Liouville, Caputo and Generalized Functions approach. For the concept of fractional derivative, we will adopt Caputo's 
definition, which is a modification of the Riemann-Liouville definition and has the advantage of dealing properly with initial value problems. For more details and some of their applications, see $[41 ; 28]$.

THEOREM 2.1. Let $f \in L_{1}\left[t_{0}, t_{f}\right]$ and $0<\alpha<1$. The left Riemann-Liouville Fractional Integral (RLFI) and the right RLFI of order $\alpha$ of a function $f$ on $L_{1}\left[t_{0}, t_{f}\right]$ is defined as:

$$
\begin{aligned}
I_{t_{0}}^{\alpha} f(x) & :=\frac{1}{\Gamma(\alpha)} \int_{t_{0}}^{x}(x-t)^{\alpha-1} f(t) d t, \\
I_{t_{f}}^{\alpha} f(x) & :=\frac{1}{\Gamma(\alpha)} \int_{x}^{t_{f}}(t-x)^{\alpha-1} f(t) d t,
\end{aligned}
$$

for all $x \in\left[t_{0}, t_{f}\right]$ and $\Gamma($.$) is known as the Euler-Gamma function.$

We denote $I_{t}^{0} f(x)=f(x)$. Obviously, the fractional integral operators $I_{t}^{\alpha}, \alpha \geq 0$, satisfy the semigroup property $I_{t}^{\alpha} I_{t}^{\beta}=I_{t}^{\alpha+\beta}, \alpha, \beta \geq 0$.

THEOREM 2.2. The left and right Caputo fractional derivatives of order $\alpha \in \mathbb{R}_{+}$ are defined, respectively by:

$$
\begin{aligned}
& { }_{t_{0}}^{C} D_{t}^{\alpha} f(x):=\frac{1}{\Gamma(n-\alpha)} \int_{t_{0}}^{x} \frac{f^{(n)}(t)}{(x-t)^{n-\alpha-1}} d t, \\
& { }_{t}^{C} D_{t_{f}}^{\alpha} f(x):=\frac{(-1)^{n}}{\Gamma(n-\alpha)} \int_{x}^{t_{f}} \frac{f^{(n)}(t)}{(t-x)^{n-\alpha-1}} d t,
\end{aligned}
$$

where $t_{0} \leq x \leq t_{f}, f^{(n)}(t)=\frac{d^{n} f(t)}{d t^{n}} \in C^{n}\left[t_{0}, t_{f}\right]$ is the ordinary derivative of integer order $n$ and $n=[\alpha]+1$. Indeed, Caputo fractional derivatives of order $\alpha, n-1<\alpha \leq n$, of function $f \in C[a, b]$ is given by:

$$
{ }^{C} D^{\alpha} f(x)=J^{m-\alpha}\left(\frac{d^{m}}{d x^{m}} f(x)\right)
$$

where $m \in \mathbb{N}$ and $m-1 \leq \alpha \leq m$.

The following theorem, helps us to apply a fractional integral over a fractional derivative [28]:

THEOREM 2.3. Let $\alpha>0$ and $n=\lceil\alpha\rceil$. If $f(x) \in C^{n}[a, b]$, then we have:

$$
I_{t_{0}}^{\alpha}\left({ }_{t_{0}}^{C} D_{t}^{\alpha} f\right)(x)=f(x)-\sum_{k=0}^{n-1} \frac{f^{(k)}\left(t_{0}\right)}{k !}\left(x-t_{0}\right)^{k},
$$




$$
I_{t_{f}}^{\alpha}\left({ }_{t}^{C} D_{t_{f}}^{\alpha} f\right)(x)=f(x)-\sum_{k=0}^{n-1} \frac{(-1)^{k} f^{(k)}\left(t_{f}\right)}{k !}\left(t_{f}-x\right)^{k} .
$$

In particular, if $0<\alpha<1$ and $f(x) \in C[a, b]$, we will have the following properties:

$$
I_{t_{0}}^{\alpha}\left[{ }_{t_{0}}^{C} D_{t}^{\alpha} f(t)\right]=f(t)-f\left(t_{0}\right),
$$

and

$$
I_{t_{f}}^{\alpha}\left[{ }_{t}^{C} D_{t_{f}}^{\alpha} f(t)\right]=f(t)-f\left(t_{f}\right) .
$$

\subsection{Adomian Decomposition Method}

The fractional ADM, has been shown to solve effectively, easily, and accurately a large class of nonlinear problems with approximations converging rapidly to accurate solutions. To illustrate its basic idea of the technique, we consider the following nonlinear fractional dynamic system:

$$
{ }_{t_{0}}^{C} D_{t}^{\alpha} x(t)+L[x(t)]+N[x(t)]=f(t), \quad x\left(t_{0}\right)=x_{0},
$$

where $L$ is a linear operator, $N$ is a nonlinear operator, $f(t)$ is a given continuous function and $0<\alpha<1$. By applying a proper fractional integral operator to (10) and using Theorem 2.3, we have:

$$
x(t)=x_{0}+g(t)-I_{t_{0}}^{\alpha}(L[x(\tau)]+N[x(\tau)]),
$$

where $g(t)$ represents a function that arising from fractional integration of $f(t)$ and using the initial condition. The basic character of the ADM is to consider $x(t)=$ $\sum_{n=0}^{\infty} x_{n}(t)$ as a correction functional for system (11), where $x_{n}$ is the $n^{\text {th }}$ approximate solution of $x$. Also, ADM requires to determine $N[x(t)]=\sum_{n=0}^{\infty} A_{n}$, in which:

$$
A_{n}=\frac{1}{n !}\left[\frac{d^{n}}{d \lambda^{n}} N[x(\lambda)]\right]_{\lambda=0} .
$$

Now, the successive approximations $x_{n+1}, n \geq 0$, of the solution $x$ will be readily obtained by substituting the approximated functions $x(t)$ and $N[x(t)]$ into (11):

$$
x_{0}(t)=x_{0}+g(t), \quad x_{n+1}(t)=-I_{t_{0}}^{\alpha}\left(L\left[x_{n}(\tau)\right]+A_{n}\right), n \geq 0 .
$$

The following theorem, helps us to apply a correction functional over a fractional differential equation.

THEOREM 2.4. Let the nonlinear term $N(x(t))$ be Lipschitz continuous with $|N(x)-N(\hat{x})| \leq R_{1}|x(t)-\hat{x}(t)|$ and also the linear term $L(x(t))$ be Lipschitz 
continuous with $|L(x)-L(\hat{x})| \leq R_{2}|x(t)-\hat{x}(t)|$, where $R_{1}$ and $R_{2}$ are Lipschitz constants. Then, the correction functional for FDE (10), that is given via the iterative formula (13), is converges if $0<\gamma<1$ and $\left|x_{1}(t)\right|<\infty$ where $\gamma=\left(R_{1}+R_{2}\right) \frac{\left(t_{f}-t_{0}\right)^{\alpha}}{\Gamma(\alpha+1)}$.

PROOF. See [11; 22] for proofs and other details.

The main step of MADM is to characterize the function $g$ in (13), by two distinct parts: one part assigned to the initial term $x_{0}$ and the other to the second term $x_{1}$ that we consider here. Please note that, this variation in the formation of $x_{0}$ and $x_{1}$ is slight, but it plays a major role in accelerating the convergence of the resulting successive approximations. Since the only difference between the ADM and MADM is on the components $x_{0}$ and $x_{1}$, so they have the same iteration formulas and the same convergence. Therefore, by applying the linearity property of operators $L$, the recursive relation (13) can be converted to the following iterative formula:

$$
\begin{aligned}
& x_{0}(t)=x_{0} \\
& x_{1}(t)=g(t)-I_{t_{0}}^{\alpha}\left(L\left[x_{0}(\tau)\right]+A_{0}\right) \\
& x_{2}(t)=-I_{t_{0}}^{\alpha}\left(L\left[x_{1}(\tau)\right]+A_{1}\right) \\
& \vdots \\
& x_{n+1}(t)=-I_{t_{0}}^{\alpha}\left(L\left[x_{n}(\tau)\right]+A_{n}\right), n \geq 1 .
\end{aligned}
$$

By using the above recurrence relation, we obtain $x_{n}(t)$ which is the $n^{t h}$ approximate solution of $x$. Since a solution containing an infinite sum is hardly practical, assuming that $N \in \mathbb{N}$ is a large number and approximate the practical solution by the following truncated series:

$$
X_{N}(t)=\sum_{n=0}^{N-1} x_{n}(t)
$$

consequently, the solution is:

$$
x(t)=\lim _{N \rightarrow \infty} X_{N}(t) .
$$

In other words, the correction functional (11) will give several approximations, and therefore the exact solution is obtained at the limit of the resulting successive approximations. 


\section{NUMERICAL SCHEME FOR SOLVING FOCPS}

Here we define the FOCP in terms of the left Caputo Fractional Derivative (CFDs) which are given in the last section. A FOCP is the problem of finding an optimal control $u(t)$ that minimizes the cost functional:

$$
\min J(u)=\frac{1}{2} \int_{t_{0}}^{t_{f}}\left\{x^{T}(t) Q(t) x(t)+u^{T}(t) R(t) u(t)\right\} d t
$$

which satisfies the following FDE:

$$
{ }_{t_{0}}^{C} D_{t}^{\alpha} x(t)=A(t) x(t)+B(t) u(t),
$$

and the initial condition:

$$
x\left(t_{0}\right)=x_{0} .
$$

where $x(t)$ is the state variable, $u(t)$ is the control variable, $Q(t)$ and $R(t)$ are chosen to be positive semidefinite and positive definite matrices respectively.

Suppose that $H(x(t), u(t), \lambda(t), t)$ is Hamiltonian of the above problem defined as:

$$
\begin{aligned}
& H(x(t), u(t), \lambda(t), t)=\frac{1}{2}\{\left.x^{T}(t) Q(t) x(t)+u^{T}(t) R(t) u(t)\right\}+ \\
&+\lambda^{T}\{A(t) x(t)+B(t) u(t)\}
\end{aligned}
$$

where $\lambda \in \mathbb{R}^{n}$ is the Lagrange multiplier also known as a co-state or an adjoint variable. Now we will find the optimal value of $x(t)$ and $u(t)$ in a way which the function $J(u)$ in problem (16) becomes minimum. The necessary conditions for optimality fractional optimal control problem are given as (see [9]):

$$
\begin{gathered}
{ }_{t_{0}}^{C} D_{t}^{\alpha} x(t)=\frac{\partial H}{\partial \lambda}=A(t) x(t)+B(t) u(t), x\left(t_{0}\right)=x_{0}, \\
\frac{\partial H}{\partial u}=R(t) u(t)+B^{T}(t) \lambda(t)=0, \\
{ }_{t}^{C} D_{t_{f}}^{\alpha} \lambda(t)=\frac{\partial H}{\partial x}=Q(t) x(t)+A^{T}(t) \lambda(t), \lambda\left(t_{f}\right)=0 .
\end{gathered}
$$

The above equations coincide with the classical ones as $\alpha$ approaches to 1 . It should 
be mentioned that in practice, we obtain:

$$
u(t)=-R^{-1} B^{T} \lambda
$$

So, it can be demonstrated that he necessary conditions for system (16)-(18) are as follows:

$$
\begin{gathered}
{ }_{t_{0}}^{C} D_{t}^{\alpha} x(t)=A(t) x(t)-B(t) R(t)^{-1} B(t)^{T} \lambda(t), x\left(t_{0}\right)=x_{0}, \\
{ }_{t}^{C} D_{t_{f}}^{\alpha} \lambda(t)=Q(t) x(t)+A^{T}(t) \lambda(t), \lambda\left(t_{f}\right)=0 .
\end{gathered}
$$

Since the initial state $x_{0}(t)$ is always known, we make an initial guess for $\lambda_{0}(t)$ that can be considered as an unknown constant. Now, by using the MADM for equations (30)-(31), approximate solutions $x_{n}(t)$ and $\lambda_{n}(t)$ will be readily obtained upon using any selective functions $x_{0}(t)$ and $\lambda_{0}(t)$ for initialization. Substituting these value into the corresponding function $u(t)$ (i.e. relation (23)), we get the final approximated solution of problem (16)-(18).

\section{NUMERICAL EXAMPLE}

In this section some examples are provided to illustrate the efficiency and applicability of the present idea.

EXAMPLE 4.1. As a first example, consider the following time-invariant FOCP which minimizes the quadratic performance index:

$$
\min J(u)=\frac{1}{2} \int_{0}^{1}\left(x^{2}(t)+u^{2}(t)\right) d t
$$

subject to the following dynamical system:

$$
{ }_{0}^{C} D_{t}^{\alpha} x(t)=-x(t)+u(t)
$$

and the boundary condition $x(0)=1$.

The exact solution of the above mentioned problem in the case of $\alpha=1$ is as following [10]:

$$
x(t)=\cosh (\sqrt{2} t)+\beta \sinh (\sqrt{2} t)
$$

and,

$$
u(t)=(1+\beta \sqrt{2}) \cosh (\sqrt{2} t)+\beta \sqrt{2} \sinh (\sqrt{2} t),
$$


where:

$$
\beta=-\frac{\cosh (\sqrt{2})+\sqrt{2} \sinh (\sqrt{2})}{\sqrt{2} \cosh (\sqrt{2})+\sinh (\sqrt{2})} \cong-0.9799 .
$$

The optimal value of performance index for this problem is $J^{*}=0.1929092981$. The necessary conditions for this problem are as follows:

$$
\begin{aligned}
& { }_{0}^{C} D_{t}^{\alpha} x(t)=-x(t)-\lambda(t), x(0)=1, \\
& { }_{t}^{C} D_{1}^{\alpha} \lambda(t)=x(t)-\lambda(t), \lambda(1)=0 .
\end{aligned}
$$

By using the recurrence formula of MADM for system (30)-(31) with different values of $N$, the optimal values of cost functional $J$ will be achieved that shown in Table I. In

Table I. Optimal values of cost functional $J$ with various choices of $N$.

\begin{tabular}{|c|cccc|}
\hline$N$ & 10 & 15 & 20 & 30 \\
\hline$J^{*}$ & 0.1929833162 & 0.1929119841 & 0.1929093060 & 0.1929092982 \\
\hline
\end{tabular}

Table II we compare the obtained results of the optimal values of the cost functional $J$ using our proposed method with those obtained approaches in the literature.

Table II. Optimal values of $J$ at different choices of $\alpha$.

\begin{tabular}{|c|ccc|}
\hline$\alpha$ & {$[12]$} & {$[4]$} & our approach \\
\hline \hline 1 & 0.192909 & 0.192909 & 0.192909 \\
0.99 & 0.19153 & 0.19153 & 0.19155 \\
0.9 & 0.17952 & 0.17953 & 0.17962 \\
0.8 & 0.16729 & 0.16711 & 0.16740 \\
\hline
\end{tabular}

EXAMPLE 4.2. Consider the following FOCP:

$$
\min J(u)=\frac{1}{2} \int_{0}^{1}\left\{\left(x(t)-t^{2}\right)^{2}+(u(t)-t+1)^{2}\right\} d t
$$

in which:

$$
{ }_{0}^{C} D_{t}^{\alpha} x(t)=\frac{\Gamma(3)}{\Gamma(2)}(x(t)-t u(t)), \quad x(0)=0 .
$$

The exact solution of this equation is given by $x(t)=t^{2}, u(t)=t-1$ when $\alpha=1$. In Figure 1, we present the accuracy of approximation state and control by MADM 
with $N=30$ and different values of $\alpha$. It is shown that as the value of $\alpha$ approaches 1 , the numerical solutions for FOCP (32)-(33) approach the analytical solutions.
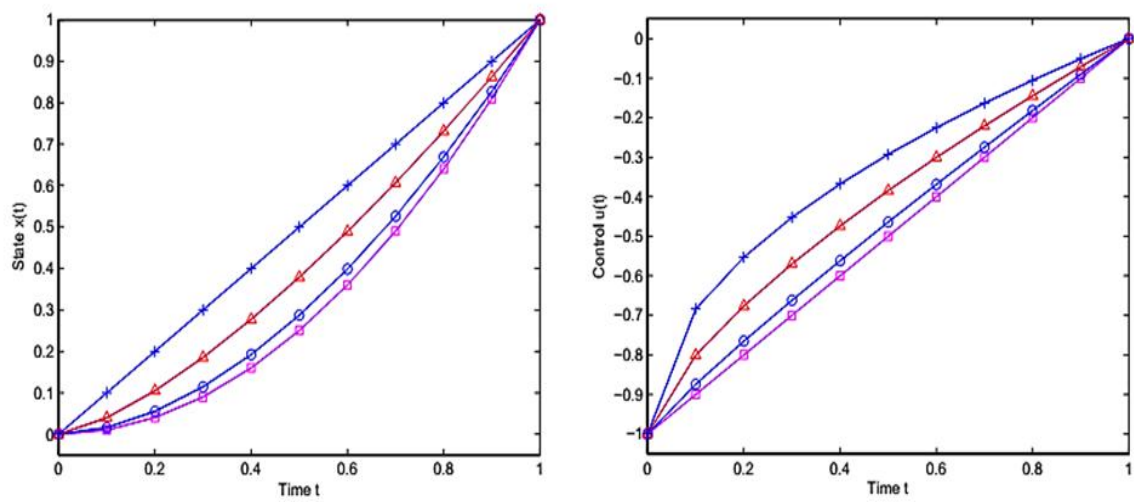

Fig. 1. Approximate solutions of $x(t)$ and $u(t)$ for different values of $\alpha$ and $N=30(\square: \alpha=1, \circ: \alpha=0.9$, $\triangle: \alpha=0.7,+: \alpha=0.5)$.

Table III shows the maximum absolute errors of the state variable $x(t)$ and control variable $u(t)$ obtained using MADM at $\alpha=0.5$ and various choices of $N$. It can be

Table III. Absolute errors of $x(t)$ and $u(t)$ at $\alpha=0.5$ and various choices of $N$.

\begin{tabular}{|c|ccc|cc|}
\hline & \multicolumn{3}{|c|}{$x(t)$} & $u(t)$ & \\
\hline$t$ & $N=20$ & $N=30$ & $N=40$ & $N=10$ & $N=20$ \\
\hline \hline 0.1 & 0 & 0 & 0 & 0 & 0 \\
0.2 & $2.7756 E-17$ & 0 & 0 & 0 & 0 \\
0.3 & $2.8310 E-15$ & 0 & 0 & 0 & 0 \\
0.4 & $1.9756 E-13$ & 0 & 0 & 0 & 0 \\
0.5 & $5.8985 E-12$ & 0 & 0 & 0 & 0 \\
0.6 & $1.0253 E-10$ & 0 & 0 & 0 & 0 \\
0.7 & $1.2226 E-9$ & $2.2204 E-16$ & 0 & 0 & 0 \\
0.8 & $1.1031 E-8$ & $4.9960 E-15$ & 0 & 0 & 0 \\
0.9 & $8.0224 E-8$ & $9.0261 E-14$ & 0 & $2.2204 E-16$ & $2.2204 E-16$ \\
\hline
\end{tabular}

seen, with increasing the number iterations $N$, the approximate solutions coincide with the exact solutions. 


\section{REFERENCES}

Alam, K. N., Uddin, K. N., Asmat, A., and Muhammad, J. (2012). Approximate analytical solutions of fractional reaction-diffusion equations. J King Saud Unive Sci., 24, 111-118.

Arikoglu, A., and Ozkol, I. (2007). Solution of a fractional differential equations by using differential transform method. Chaos Solit Fractals, 34, 1473-1481.

Alipour, M., Rostamy, D. and Baleanu, D. (2013). Solving multi-dimensional fractional optimal control problems with inequality constraint by Bernstein polynomials operational matrices. Journal of Vibration and Control, 19, 2523-2540.

Alizadeh, A., and Effati, S. (2016). An iterative approach for solving fractional optimal control problems. Journal of Vibration and Control, 8, 1-19.

Adomian, G. (1989). Nonlinear Stochastic Systems Theory and Applications to Physics. Kluwer Academic Publishers Kluwer.

Adomian, G. (1988). A review of the decomposition method in applied mathematics. Journal of Mathematical Analysis and Applications, 135, 501-544.

Adomian, G. (1991). Solving frontier problems modelled by nonlinear partial differential equations. Computers and Mathematics with Applications, 22, 91-94.

Ahmed, E., and Elgazzar, A. S. (2007). On fractional order differential equations model for nonlocal epidemics. Physica A, 379, 607-614.

Agrawal, O. P. (2004). A general formulation and solution scheme for fractional optimal control problems. Nonlinear Dynamics, 38, 323-337.

Agrawal, O. P. (2008). A Formulation and Numerical Scheme for Fractional Optimal Control Problems. Journal of Vibration and Control, 14, 1291-1299.

Abbaoui, K., and Cherruault, Y. (1994). Convergence of Adomian Method Applied to Nonlinear Equations. Mathematical and Computer Modelling, 20, 69-73.

Akbarian, T., and Keyanpour, M. (2013). A New Approach to the Numerical Solution of Fractional Order Optimal Control Problems. Applications and Applied Mathematics, 8, 523-534.

Bhrawy, A. H., Doha, E. H., Machado, J. A. T. and Ezz-Eldien, S. S. (2015). An efficient numerical scheme for solving multi-dimensional fractional optimal control problems with a quadratic performance index. Asian Journal of Control, 18, 1-14.

Bohannan, G. W. (2008). Analog fractional order controller in temperature and motor control applications. Journal of Vibration and Control, 14, 1487-1498.

Changpin, Li., and Yihong, W. (2009). Numerical algorithm based on Adomian decomposition for fractional differential equations. Comput Math Appl, 57, 1672-1681.

Cole, K. S. (1993). Electric conductance of biological systems. in: Proc. Cold Spring Harbor Symp. Quant. Biol, Cold Spring Harbor, New York, 107-116.

Duan, J., Jianye, An., and Mingyu, Xu. (2007). Solution of system of fractional differential equations by Adomian decomposition method. Appl Mathematics-A J Chin Univ, 22,7-12.

Duan, J., Temuer, C., Randolph, R., and Lei, L. (2013). The Adomian decomposition method with convergence acceleration techniques for nonlinear fractional differential equations. Comput Math Appl, $66,728-736$. 
Das, S. (2009). Analytical solution of a fractional diffusion equation by variational iteration method. Comput Math Appl, 57, 483-437.

Duan, J.S ., Rach, R., Baleanu, D., and Wazwaz A. M. (2012). A review of the Adomian decomposition method and its applications to fractional differential equations. Commun. Fractional Calculus, 3, 73-99. Erturk, V. S., and Momani, S. (2008). Solving systems of fractional differential equations using differential transform method. J Comput Appl Math, 215, 142-151.

Hosseini, M. M., and Nasabzadeh, H. (2006). On the convergence of Adomian decomposition method. Applied Mathematics and Computation, 182, 536-543.

Hilfer, R. (2000). Applications of fractional calculus in physics. Singapore, Word Scientific Company. Inc, M. (2008). The approximate and exact solutions of the space- and time- fractional Burger's equations with initial conditions by variational iteration method. J Math Anal Appl, 345, 476-484.

Jesus, I. S., Machado, J. A. T., and Cunha J. B. (2008). Fractional electrical impedances in botanical elements. Journal of Vibration and Control, 14, 1389-1402.

Jesus, I. S., Machado, J. A. T., and Cunha J. B. (2006). Fractional order electrical impedance of fruits and vegetables. in: Proceedings of the 25th IASTED International Conference MODELLING, IDENTIFICATION, AND CONTROL, February 6-8, Lanzarote, Canary Islands, Spain.

Jiang, Y., and Ma, J. (2011). Higher order finite element methods for time fractional partial differential equations. J Comput Appl Math, 235, 3285-3290.

Kilbas, A. A. A., Srivastava, H. M., and Trujillo J. J. (2006). Theory and applications of fractional differential equations. (Vol. 204). Elsevier Science Limited.

Liu, J., and Hou, G. (2011). Numerical solutions of the space and time fractional coupled Burgers equation by generalized differential transform method. Appl Math Comput, 217, 7001-7008.

Momani, S., and Odibat, Z. (2007). Numerical approach to differential equations of fractional orders. $J$ Comput Appl Math, 207,96-110.

Meerschaert, M., and Tadjeran, C. (2006). Finite difference approximations for two sided space fractional partial differential equations. Appl Numer Math, 56, 80-90.

Nemati, A., and Yousefi, S.A. (2016). A Numerical Method for Solving Fractional Optimal Control Problems Using Ritz Method. Journal of Computational and Nonlinear Dynamics, 11, 051015-1 051015-7.

Nigmatullin, R. R., and Nelson, S.O. (2006). Recognition of the fractional kinetics in complex systems: Dielectric properties of fresh fruits and vegetables form 0.01 to1.8 GHz. Signal Processing, 86, 2744 2759.

Odibat, Z., and Momani, S. (2008). Numerical methods for nonlinear partial differential equations of fractional order. Appl Math Model, 32, 28-39.

Odibat, Z., Momani, S., and Erturk, V. S. (2008). Generalized differential transform method: application to differential equations of fractional order. Appl Math Comput, 197,467-477.

Pandey, R. K., Singh, O. P., and Baranwal, V. K. (2011). An analytic algorithm for the space-time fractional advectionedispersion equation. Comput Phys Commun, 182, 1134-44.

Petrovic, L. M., Spasic, D. T., and Atanackovic, T. M. (2005). On a mathematical model of a human root dentin. Dental Materials, 21, 125-128 . 
Ray, S. S., and Bera, R. K. (2005). Analytical solution of BagleyeTorvik equation by Adomian decomposition method. Appl Math Comput, 168, 398-410.

Saadatmandi, A., and Dehghan, M. (2010). A new operational matrix for solving fractional order differential equations. Comput Math Appl, 59, 1326-36.

Surez, J. I., Vinagre, B. M., and Chen, Y. (2008). A fractional adaptation scheme for lateral control of an AGV. Journal of Vibration and Control, 14, 1499-1511.

Samko, S. G., Kilbas, A. A., and Marichev O. I. (1993). Fractional integrals and derivatives. Theory and Applications, Gordon and Breach, Yverdon.

Wazwaz, A. M., and El-Sayed, S. M. (2001). A new modification of the Adomian decomposition method for linear and nonlinear operators. Applied Mathematics and Computation, 122, 393-405.

Zamani, M., Karimi-Ghartemani, M., and Sadati, N. (2007). FOPID controller design for robust performance using particle swarm optimization. Fractional Calculus and Applied Analysis, 10, 169-187.

\author{
Samaneh Soradi Zeid \\ Department of Mathematics, Faculty of Mathematics \\ University of Sistan and Baluchestan, Zahedan, Iran \\ email: s_soradi@yahoo.com
}

Mostafa Yousefi

National Iranian Oil Products Distribution Company (NIOPDC)

Zahedan Region, Zahedan, Iran

email: mostafayousefi12@gmail.com

Morteza Yousefi

National Iranian Gas Company (NIGC)

Zahedan Region, Zahedan, Iran 